\title{
A 40-550 MHz Harmonic-Free All-Digital Delay-Locked Loop Using a Variable SAR Algorithm
}

\author{
Rong-Jyi Yang, Student Member, IEEE, and Shen-Iuan Liu, Senior Member, IEEE
}

\begin{abstract}
A wide-range all-digital delay-locked loop (ADDLL) is presented to achieve low jitter, low power and process immunity. The variable successive approximation register-controlled algorithm is proposed to eliminate the harmonic-locking issue in wide-range operation. It can also achieve the fast-locking property and closed-loop operation. With the balanced edge combiner, the ADDLL outputs a synchronous clock with the duty cycle close to $50 \%$ when the duty cycle of the input clock varies from $20 \%$ to $80 \%$. Fabricated in $0.18 \mu \mathrm{m}$ CMOS technology, the ADDLL maintains a fixed one input clock cycle latency from $40 \mathrm{MHz}$ to $550 \mathrm{MHz}$ without the harmonic-locking issue. It dissipates $12.6 \mathrm{~mW}$ from a $1.8 \mathrm{~V}$ supply at $550 \mathrm{MHz}$. The measured root-mean-square and peak-to-peak jitters at $550 \mathrm{MHz}$ are $1.5 \mathrm{ps}$ and $12 \mathrm{ps,}$, respectively.
\end{abstract}

Index Terms-ADDLL, delay-locked loop (DLL), DCC, edge combine, harmonic lock, successive approximation register (SAR), variable successive approximation register (VSAR).

\section{INTRODUCTION}

D ELAY-LOCKED loops (DLLs) are widely used as de-skew buffers and clock generators in microprocessors [1], DSPs, multi-core SoCs [2], DRAM interfaces [4]-[8] and application-specified integrated circuits. To meet the various specifications for different applications, it is better for a DLL to achieve low cost, wide operating frequency range, low jitter performance, small power consumption and process immunity. Conventional analog approaches achieve better performances in jitter and skew but the process-sensitive characteristic makes them difficult to migrate to advanced technologies. On the contrary, the digital DLL is a better choice due to the compatibility for system integration and the insensitivity to the supply noise.

Digital DLLs are roughly divided into three categories. The first one adopts the sequential search algorithm, i.e., the countercontrolled scheme [5], [7], [8]. The phase detector (PD) for a counter-controlled DLL is a simple D-type flip-flop (DFF) but the lock time increases exponentially as the number of control bits increases. The second one adopts the flash architecture, i.e., the time-to-digital (TDC) scheme [9]. Large chip area and power are required to achieve the shortest lock time. The last one adopts the binary search algorithm, i.e., the successive approximation register-controlled (SAR) scheme [10]. The SAR scheme seems to be a better choice in considerations of the lock time and the hardware complexity. However, its open-loop characteristic does not track the process, voltage, temperature, and

Manuscript received March 15, 2006; revised September 1, 2006. This work was supported in part by MediaTek Inc. and the National Science Council, Taiwan.

The authors are with the Graduate Institute of Electronics Engineering and Department of Electrical Engineering, National Taiwan University, Taipei, Taiwan 10617, R.O.C. (e-mail: 1si@cc.ee.ntu.edu.tw).

Digital Object Identifier 10.1109/JSSC.2006.889381 loading (PVTL) variations [11]. For a conventional SAR DLL in wide-range applications, the harmonic-locking issue occurs and the output clock jitter increases due to the longer delay line [6], [8]. Further, the harmonic lock is not allowable in the multiphase applications and an additional detection circuit is required [12].

A 50\% duty cycle is often required in double-edge-triggered applications. The dual-loops architecture [7] and the complementary dual-structures [11], [13] are often adopted to realize the duty cycle requirement but the hardware overhead is almost double. The setting/resetting architecture [12] with an edge combiner achieves $50 \%$ duty cycle by using single delay line. However, the delay mismatch between the setting and resetting paths causes the duty cycle distortion especially for high frequencies. The delay of the edge combiner also results in the steady-state phase error.

With the proposed variable successive approximation register-controlled (VSAR) algorithm, the harmonic-locking issue is solved. The latency of the delay line is exactly one clock cycle to minimize the supply-induced jitter. The lock time is reduced as well in comparisons with the counter and the conventional SAR methods. By the proposed balanced edge combiner, the all-digital DLL accomplishes a synchronous clock with 50\% duty cycle via a single-ended reference clock and single delay line. The closed-loop characteristic is also guaranteed by transforming the VSAR controller into a counter.

This paper is organized as follows. In Section II, the design constraints and tradeoffs for the conventional SAR algorithm in wide-range applications are discussed. The proposed VSAR algorithm and the all-digital DLL are also introduced. The circuit description is given in Section III. The experimental results are illustrated in Section IV and Section V gives the conclusions of this work.

\section{The Proposed VSAR Algorithm AND THE ALL-DigITAL DLL}

The aim of the proposed VSAR algorithm is to perform binary search without the harmonic-locking issue. Fig. 1 gives the flowchart of this algorithm and the proposed VSAR controller is shown in Fig. 2. It consists of a divide-by-2 frequency divider, $M$-bit VSAR units, $(N-M)$-bit conventional SAR units, a fail-to-lock judgment circuit (FJC) and a timing control circuit, where $N$ is the total number of control bits for the digital-controlled delay line (DCDL) and $1 \leq M \leq N$. Initially, the conventional $(N-M)$-bit SAR units borrow one bit as a MSB from the LSB of the VSAR units to perform a $(N-M+1)$-bit binary search. After the binary search is done, a judgment circuit examines the lock state. Once the unlock state is detected, the conventional SAR units borrow one more LSB from the VSAR 


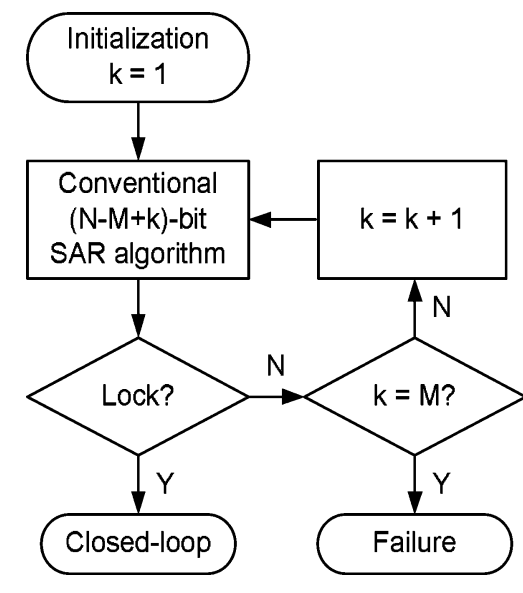

Fig. 1. Flowchart of the proposed VSAR algorithm.

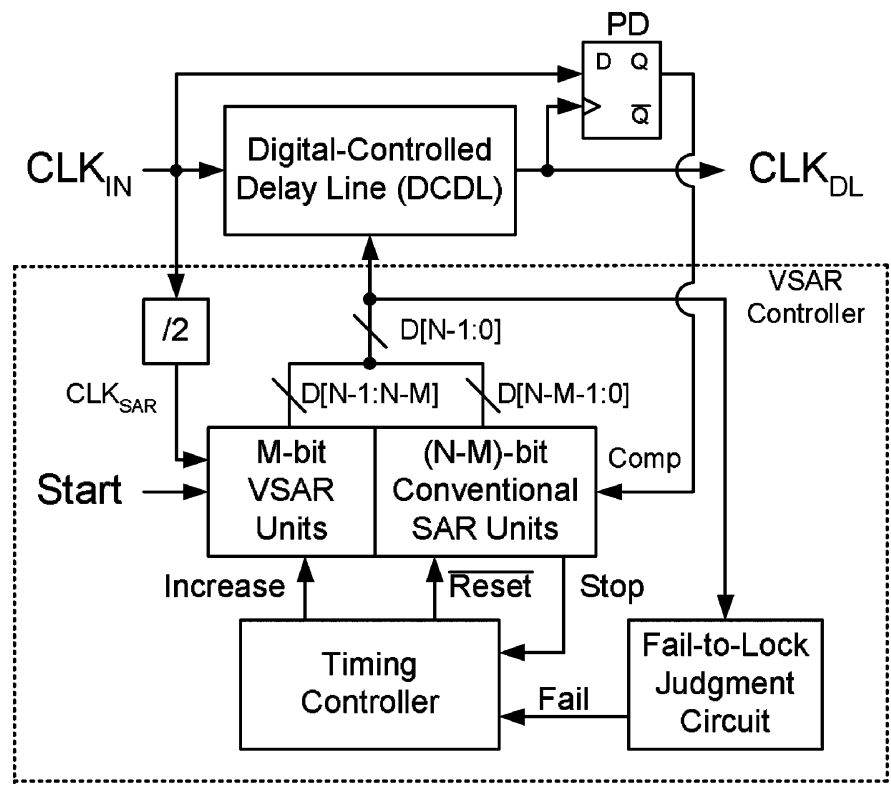

Fig. 2. Proposed VSAR controller.

units to lock again, i.e., $(N-M+2)$-bit binary search. This is equivalent to double the delay line. Before the number of borrowed bits reaches to $M$, the operation repeats until the DLL is locked correctly. Once the lock state is confirmed, the VSAR controller is transformed into a counter for a closed-loop operation. To explain the operation, a simplified timing diagram for $N=4$ and $M=3$ is illustrated in Fig. 3 . When the signal Start arises, the VSAR units lend 1 LSB to the 1-bit conventional SAR unit to perform a 2-bit binary search. The signal Stop arises while the binary search is done. If the signal Fail arises in the FJC, the fail-to-lock state is verified and the SAR circuit is reset for the next binary search. The number of lent bits of VSAR units increases by 1 and a 3-bit binary search starts. The entire operation stops if the delay corresponding to one clock period is achieved.

The VSAR algorithm seems to have a longer lock time than the conventional one. However, this may be not true. The key is the division ratio $(D R)$, which is the frequency ratio between the SAR controller clock, $\mathrm{CLK}_{\mathrm{SAR}}$, and the input clock,
CLK IN. The $D R$ for a conventional SAR controller is expressed as [14]

$$
D R>\left[T_{\mathrm{LOOP}} / T_{\mathrm{CLK}}\right]+1
$$

where [] denotes the Gaussian operation, $T_{\mathrm{CLK}}$ is the input clock period, and $T_{\mathrm{LOOP}}$ is the loop delay which includes the propagation delays of the delay line, the PD, the SAR controller and so on. For example, a delay line with a 3-bit binary code and the corresponding delay from $1 \mathrm{~ns}$ to $8 \mathrm{~ns}$ is shown in Fig. 4(a). If the input clock period is $6 \mathrm{~ns}$, the locking process using the conventional SAR algorithm is illustrated in Fig. 4(b) and the minimum $D R$ of 2 is chosen. Owing to the non-zero response time of the delay line, the code must be ready before the next rising edge of the input clock comes. Hence, the falling edge of the input clock is utilized to trigger the SAR controller. The first bit of the SAR controller switches from 0 to 1 and the rising edge of the input clock through the delay line is delayed with $5 \mathrm{~ns}$. The PD compares the phase relation between the next input clock and the output of the delay line and the output of PD goes to logic one because the rising edge of $\mathrm{CLK}_{\mathrm{DL}}$ leads that of $\mathrm{CLK}_{\mathrm{IN}}$. Hence, the first bit is fixed to logic one and the second bit of the SAR controller switches from 0 to 1 at the same time. The rising edge of the input clock is delayed with $7 \mathrm{~ns}$ at this moment. The output of PD goes to logic zero because the rising edge of $\mathrm{CLK}_{\mathrm{DL}}$ lags that of CLK $\mathrm{IN}$. Hence, the second bit goes back to logic zero. The remaining bits are determined similarly.

If the input clock period is $1 \mathrm{~ns}$, the corresponding timing diagram is shown in Fig. 4(c). The first input clock through the delay line is similarly delayed with $5 \mathrm{~ns}$, i.e., five clock cycles in this case. Any phase comparison before the input clock goes through the delay line is meaningless. It means that the SAR controller has to wait for six cycles until the output of PD is valid. The output of PD goes to logic zero because the rising edge of CLK $\mathrm{DL}$ lags that of CLK $\mathrm{IN}_{\mathrm{N}}$. Hence, the first bit goes to logic zero. Although the input clock is delayed with only $3 \mathrm{~ns}$ for the next binary search, the constraint of the $D R$ is dominated by the MSB of the binary search. For a wide-range DLL, the minimum $D R$ of 2 is not allowed and $D R$ of 6 is chosen in this case. By neglecting the delays contributed by the PD, the SAR controller and so on, $D R_{\text {wide }}$ for a wide-range DLL is expressed as

$$
D R_{\text {wide }}>\left[T_{\mathrm{DL}, \text { mid }} / T_{\mathrm{CLK}, \min }\right]+1
$$

where $T_{\mathrm{DL} \text {,mid }}$ represents the delay for the delay line controlled with the code which is half the full scale and $T_{\mathrm{CLK} \text {,min }}$ indicates the minimum input clock period in a wide-range operation. Assume the total number of bits is $N$ and the ratio between the maximum and the minimum operating frequencies is $R$. The lock time for a wide-range DLL with an $N$-bit conventional SAR controller is expressed as

$$
T_{\text {lock, CSAR }}=D R_{\text {wide }} \cdot(N+1)=\left(\left[\frac{R}{2}\right]+1\right) \cdot(N+1) .
$$

In this design, the values of $N$ and $R$ are 11 and 12, respectively. For the conventional SAR controller, $D R_{\text {wide }}$ is 7 . For the sake of convenient implementation, the practical choice is 8 . Hence, the DLL with the conventional SAR controller takes 96 cycles 


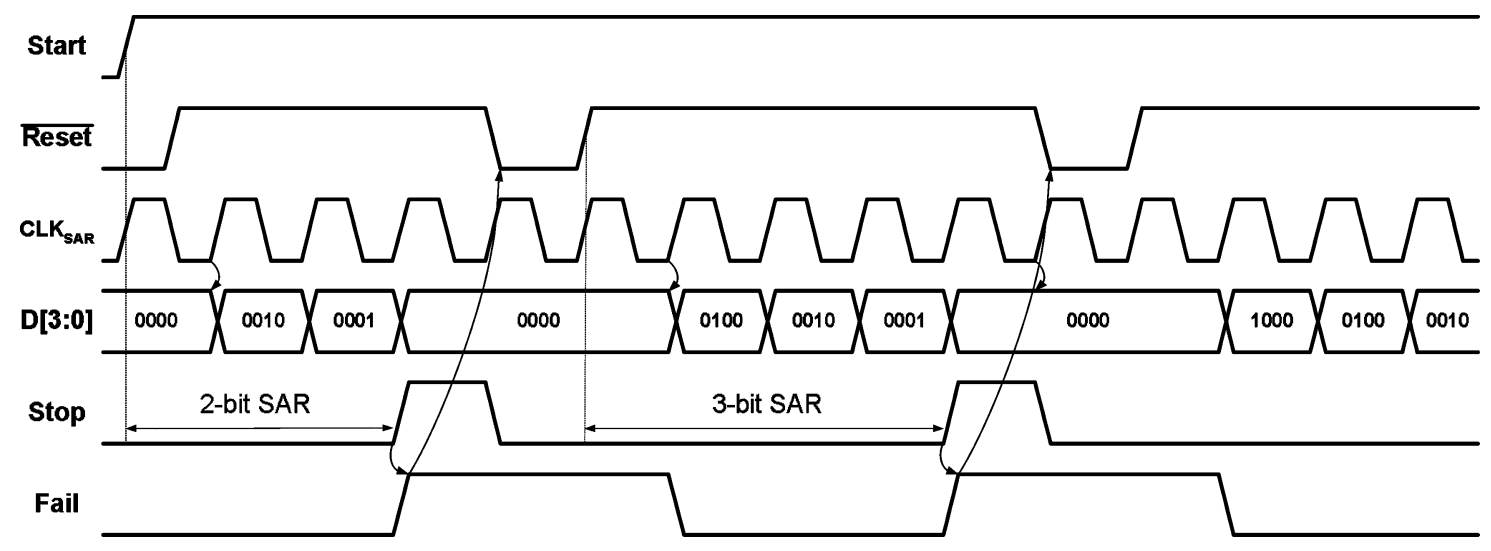

Fig. 3. Timing diagram for the VSAR algorithm while $N=4$ and $M=3$.

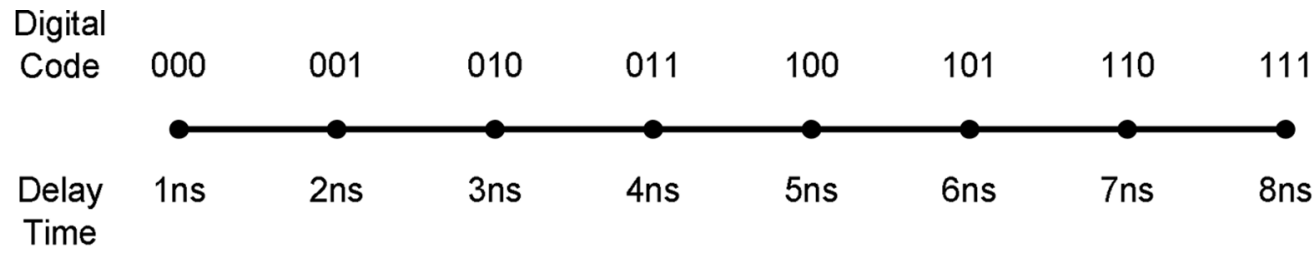

(a)

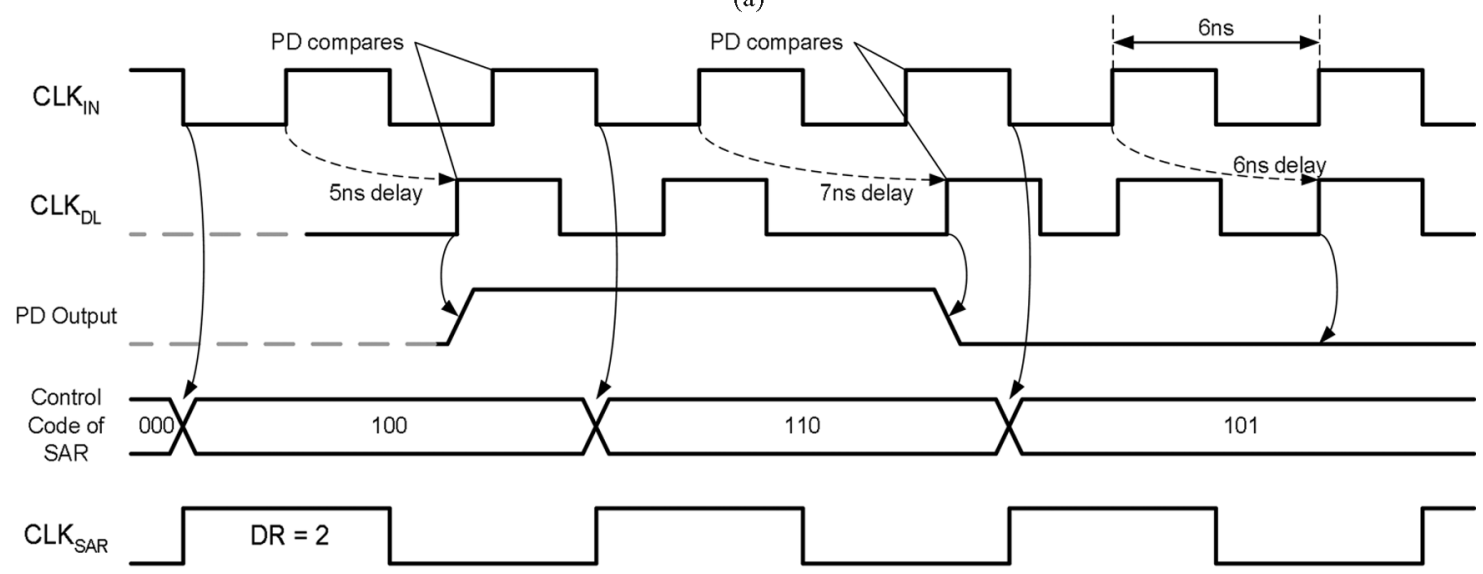

(b)

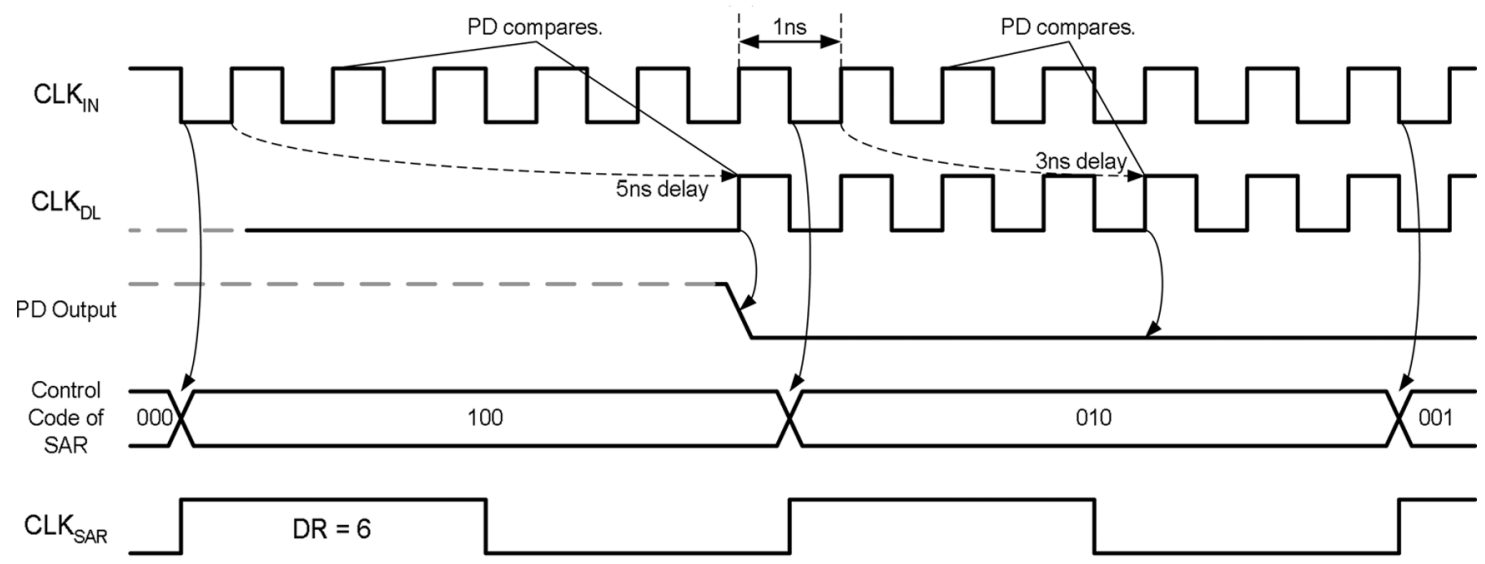

(c)

Fig. 4. (a) The 3-bit digital delay line. (b) Timing diagram when the input clock period is $6 \mathrm{~ns}$. (c) Timing diagram when the input clock period is $1 \mathrm{~ns}$. 


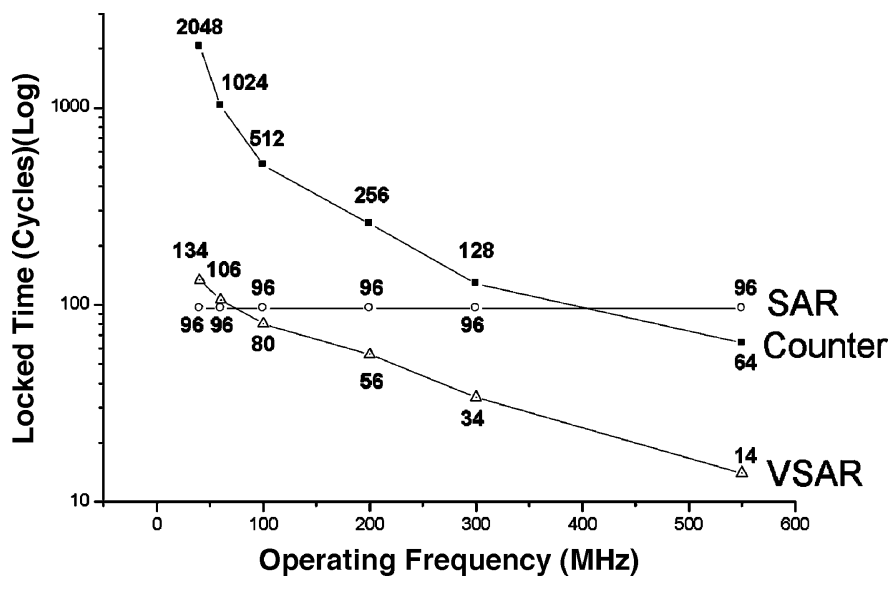

Fig. 5. Simulated lock time versus the operating frequency.

at least to lock for all frequencies, not to mention the necessary hardware for the harmonic detection.

For the VSAR controller, the delay of the delay line increases gradually from the minimum and never exceeds twice the input clock period. Thus, the harmonic locking is no longer an issue. According to (2), $D R_{\text {wide }}$ in the VSAR controller is the minimum of 2. The lock time for the DLL with the VSAR controller is given as

$$
\begin{array}{r}
T_{\text {lock }, \mathrm{VSAR}}=2 \cdot\left\{\sum_{i=N-M+1}^{k}(i+1)+2 \times[k-(N-M+1)]\right\}, \\
N-M+1 \leq k \leq N
\end{array}
$$

where $k$ is the number of bits required for correct lock. The extra $2 \times[k-(N-M+1)]$ cycles are the required time to evaluate the lock state and reset the VSAR controller before the next binary search. For example, assume that the DLL locks correctly with 8-bit binary search and the designed value of $M$ is 6 . The lock time is $2 \cdot\left[\sum_{i=6}^{8}(i+1)+2 \cdot(8-6)\right]=56$ cycles. It is shorter than that of the conventional SAR controller. The lock time increases while the number of required bits increases, i.e., the larger the clock period, the longer the lock time. The longest lock time is 2 . $\left[\sum_{i=6}^{11}(i+1)+2 \cdot(11-6)\right]=134$ cycles while the DLL locks at the lowest frequency of $40 \mathrm{MHz}$. Fig. 5 illustrates the simulated lock times for the counter-controlled, the SAR-controlled and the VSAR-controlled schemes. The proposed VSAR algorithm achieves the shortest lock time for most frequencies.

To realize a synchronous clock with 50\% duty cycle, the proposed DLL with the VSAR algorithm is shown in Fig. 6. It consists of an 11-bit VSAR controller, a PD, two DFFs, two DCDLs, an edge combiner and an 11-bit latch. The number of the variable bits in the VSAR controller is 6 , i.e., $M=6$. A 6-digit shift register, $L[5: 0]$, controls the number of lent bits. A timing diagram for the proposed DLL is illustrated in Fig. 7. The entire operation includes three phases: the period measurement phase, the synchronizing phase and the counting phase.

When the signals Lat and Count are both logic zeros, the DLL operates in the period measurement phase. The input clock period is measured via two DCDLs, i.e., the delay of each DCDL is a half of the clock period. Assume these two DCDLs are matched and the output duty cycle after the edge combiner will

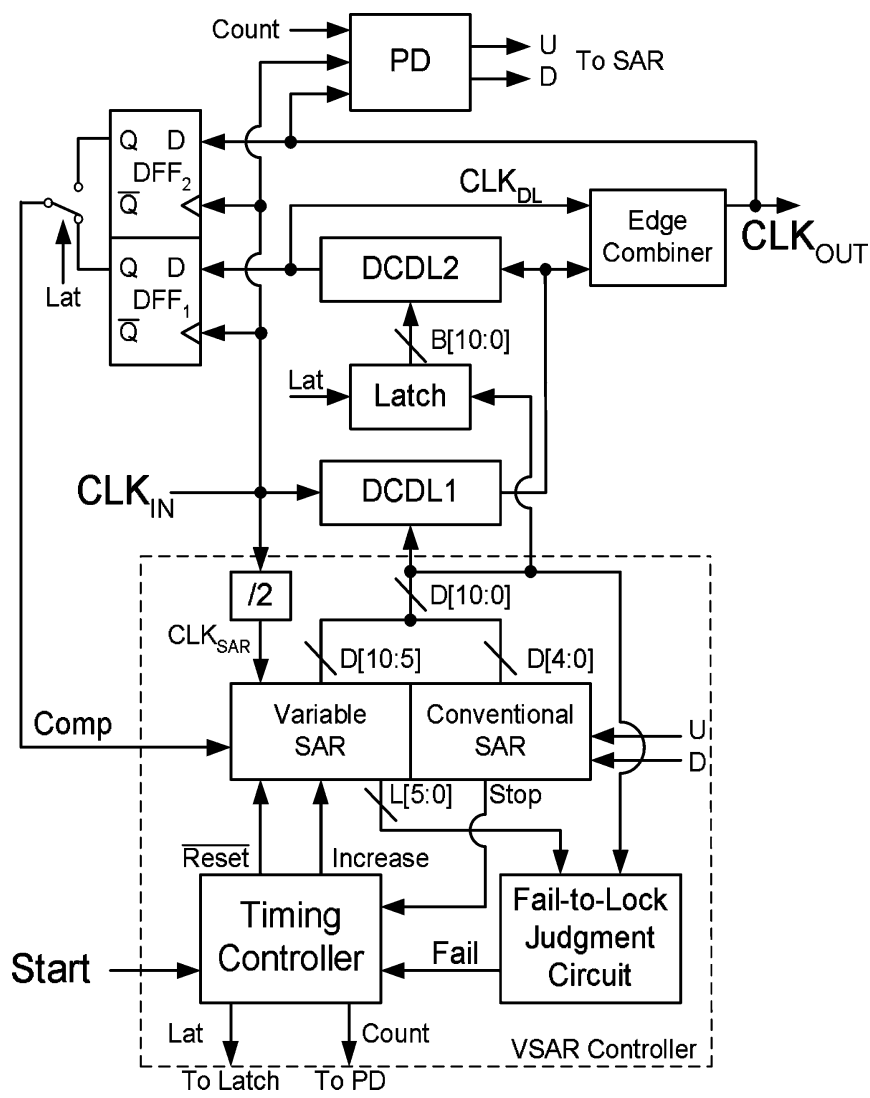

Fig. 6. Proposed all-digital DLL.

be $50 \%$. In the period measurement phase, the VSAR controller adjusts two DCDLs, i.e., $B[10: 0]$ is the same as $D[10: 0]$, according to the output of DFF1. The 6-bit binary search starts while $L[5: 0]$ is preset to 000001 where only one bit is high. If the binary search is complete and the fail-to-lock state is confirmed, i.e., the signal Fail arises while the signal Stop goes to logic one, the delay line is too short for current clock period. Then the signal, $L[5: 0]$, is shifted left to be 000010 and the SAR controller is reset for the next binary search. Once the binary search is finish and the lock state is confirmed, i.e., the signal Fail remains logic zero while the signal Stop goes to logic one, the signal Lat in the timing controller arises to indicate the end of the period measurement phase. The DLL locks correctly with one clock period. At the mean time, the digital control code, $B[10: 0]$, for the DCDL2 is latched and the delay for each DCDL is half clock period.

When the signal Lat is logic one and the signal Count is logic zero, the DLL operates in the synchronizing phase to compensate the extra delay contributed by the edge combiner. The VSAR controller only adjusts the DCDL1 according to the output of DFF2. $B[10: 0]$ is fixed while $D[10: 0]$ changes with the binary search algorithm. After the binary search is done, the DCDL1 absorbs the delay of the edge combiner and the output clock aligns with the input clock. The signal Count arises to indicate the end of the synchronizing phase.

When the signals Lat and Count are both logic ones, the DLL operates as a closed-loop to track the PVTL variations in the counting phase. The signal Count enables the PD and transforms the VSAR controller into a counter. Hence, a synchronous clock 


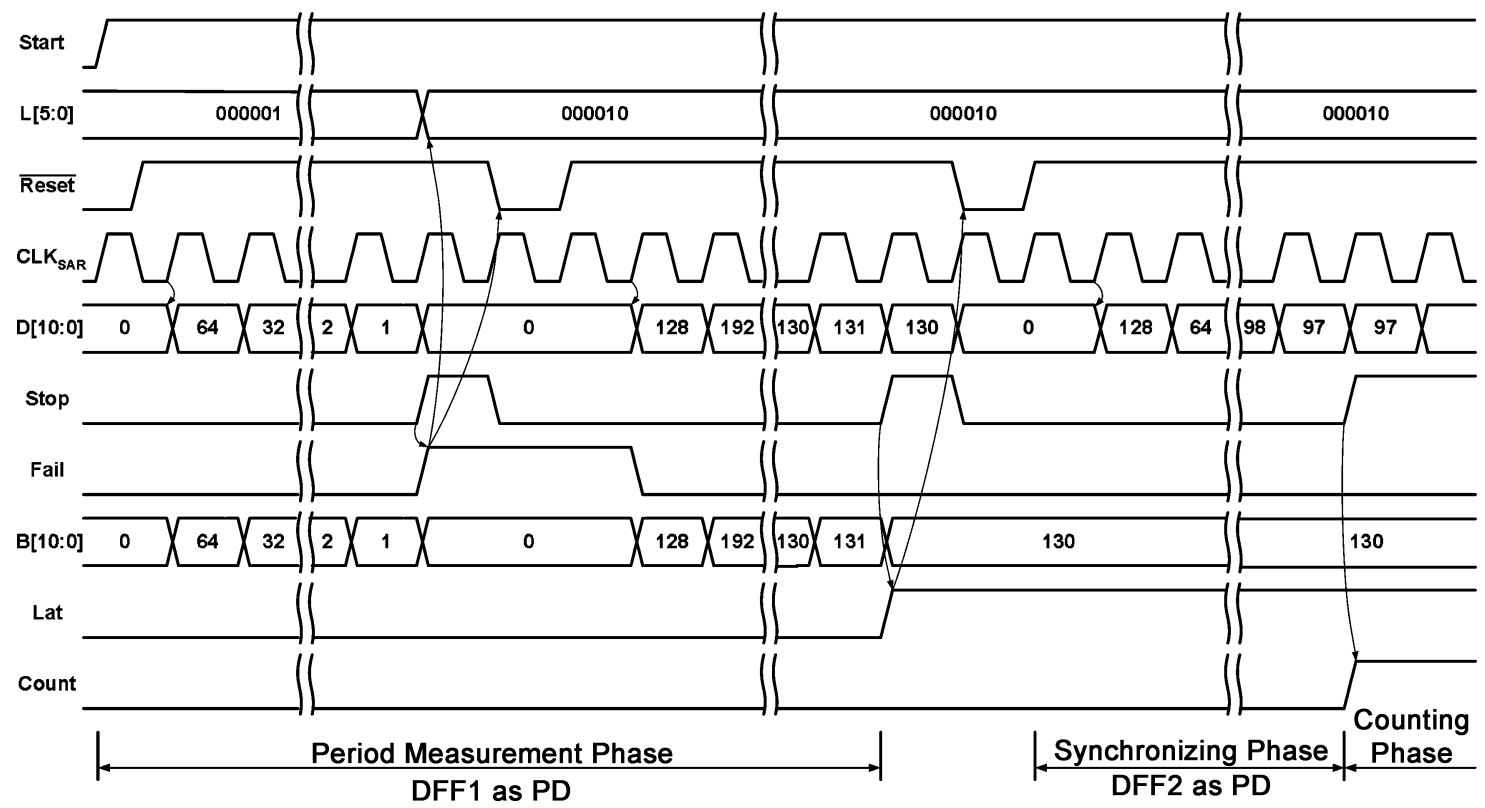

Fig. 7. Timing diagram for the proposed all-digital DLL.

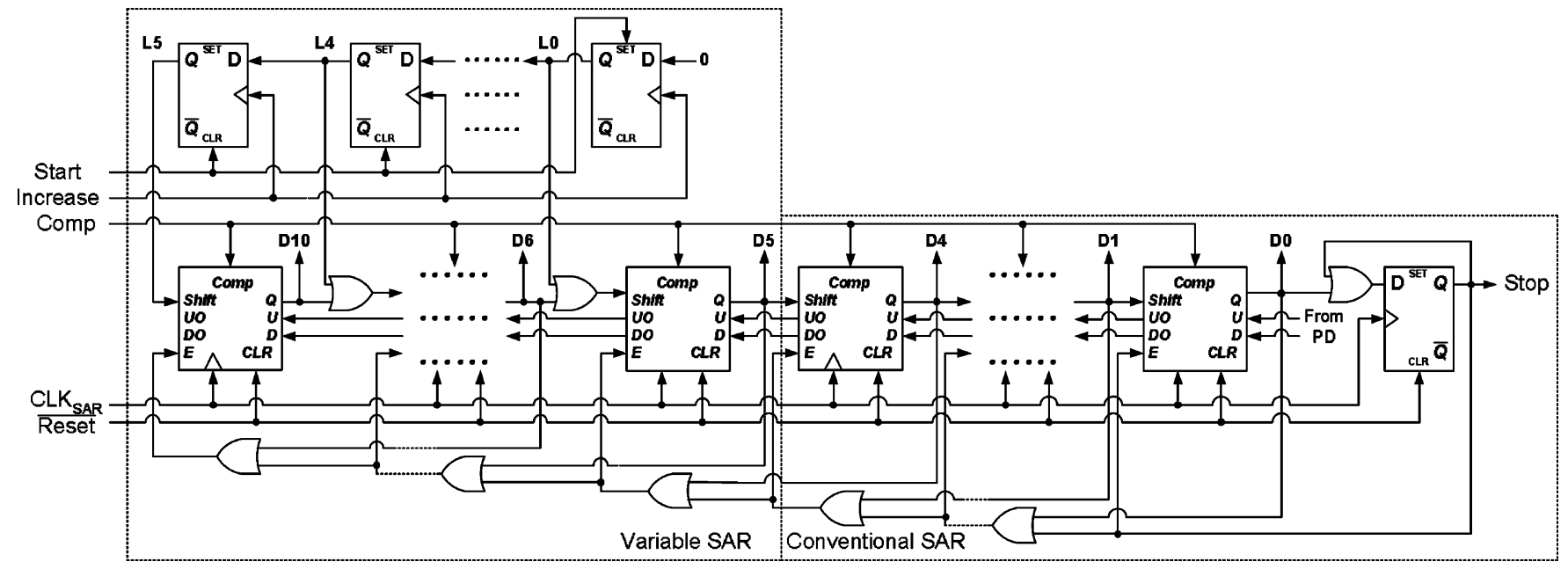

Fig. 8. The 6-bit VSAR units cascading with 5-bit conventional SAR units.

with $50 \%$ duty cycle is accomplished with a single-ended input clock and a single delay line. The accuracy of the output duty cycle still depends on the delay matching between DCDL1 and DCDL2.

\section{CirCUIT Description}

\section{A. VSAR Controller}

The proposed VSAR controller is realized by 6-bit VSAR units and 5-bit conventional SAR units as shown in Fig. 8. In the conventional SAR controller, the output $Q$ of every SAR unit is directly connected to the signal Shift of the next unit and the signal Shift of the first unit is always logic one. Hence, the number of bits in a conventional SAR controller is fixed. In the VSAR controller, one OR gate is inserted between two successive conventional SAR units. Six DFFs form a shift register. The first five DFFs from the MSB are preset to logic zero while the last one is preset to logic one. The digital code $L[5: 0]$ decides how many VSAR units are borrowed. If $L[5: 0]$ is 000010 , two bits in VSAR are borrowed and the binary search starts from the 7th bit. Hence, the length of binary search can be changed via $L[5: 0]$. To avoid the open-loop nature of the conventional SAR controller and to track the PVTL variations, the modified SAR unit illustrated in Fig. 9 is transformed into a counter unit after the binary search is complete [15]. The signals Shift and Comp for the binary search are ignored while the signal $E$ is logic one. The logic gates marked by the dash lines decide the current output according to the signals $U$ and $D$ and the corresponding carry-out signals $U O$ and $D O$ are generated as well.

\section{B. Fail-to-Lock Judgment Circuits}

Assume that the delay of a 3-bit DCDL is less than the clock period and the results of the binary search are illustrated in Fig. 10, where the labels 1, 2, 3, and 4 indicate the sequence of the digital transient process. If the middle of the delay line lies 


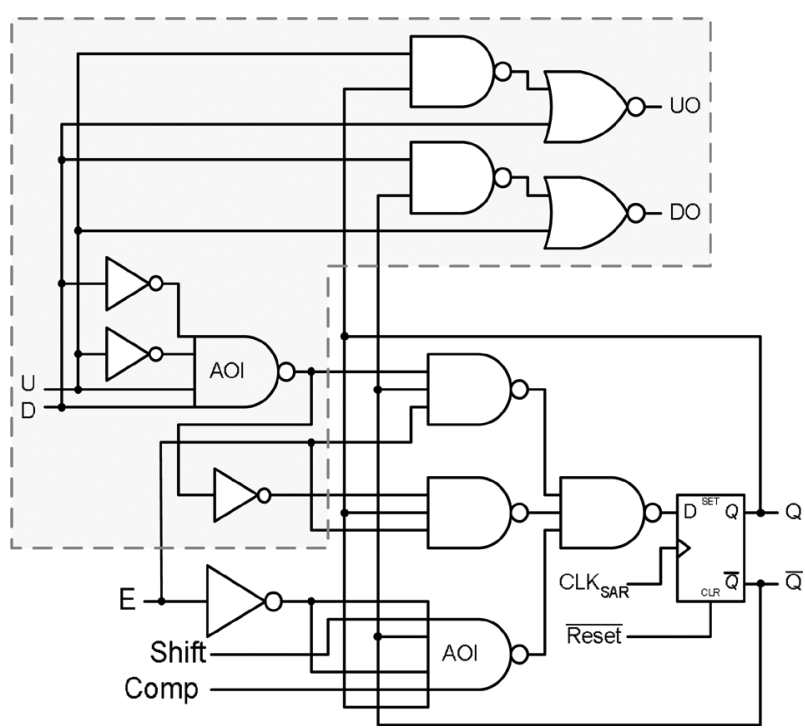

Fig. 9. Modified SAR unit.

on the positive duty of the clock, the outputs of the SAR controller after the binary search are all zeros as shown in Fig. 10(a). On the contrary, if the middle of the delay line lies on the negative duty of the clock, the outputs are all ones as shown in Fig. 10(b). The FJC can be realized with an all-ones and allzeros detector. However, there is one more thing to be noticed. Once the number of used digits is not the maximum, the outputs are not all ones. For example, if there are 6 digits used in current binary search and the fail-to-lock condition takes place, the last 6 bits from the LSB of the SAR controller are all ones and the first 5 bits from the MSB remain zeros, i.e., $D[10: 0]=$ 00000111111. The FJC for the all-ones case is relative to the number of lent bits, $L[5: 0]$. The correct logic combination for the signal Fail is represented as

$$
\begin{array}{r}
\text { Fail } \left.=\left(\left(\left(\left(L_{5} D_{10}+L_{4}\right) D_{9}+L_{3}\right) D_{8}+L_{2}\right) D_{7}+L_{1}\right) D_{6}+L_{0}\right) \\
\times D_{5} D_{4} \ldots D_{0}+\overline{D_{10}+D_{9}+\ldots+D_{0}} .
\end{array}
$$

The complete FJC is shown in Fig. 11. Finally, the timing controller generates the required control and reset signals for the VSAR controller and is shown in Fig. 12.

\section{Digital-Controlled Delay Line}

The conventional digital-controlled delay unit with two different delays controlled by a multiplexer is illustrated in Fig. 13(a). The tunable delay range increases by cascading the delay units but the intrinsic delay increases as well. To expand the operating frequency range, the number of cascaded delay units should be increased. Thus, the maximum operating frequency is restricted by the large intrinsic delay. The proposed lattice delay unit (LDU) is shown in Fig. 13(b) and the lattice delay line (LDL) constructed by cascading the LDUs is shown in Fig. 13(c). The binary control bits are converted into the thermometer codes. The tuning range increases, and the minimal delay is not changed. Both the intrinsic delay and the delay step in an LDL are the delay of two NAND gates. As the operating frequency increases, the number of activated delay units is reduced and the power consumption remains the same.

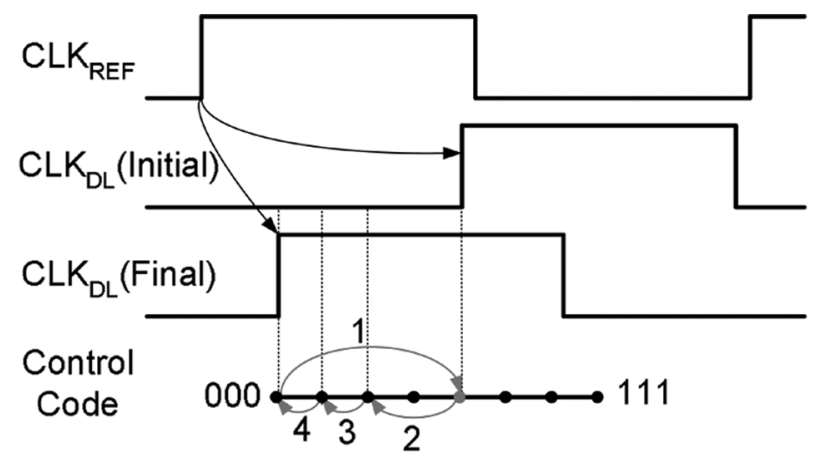

(a)

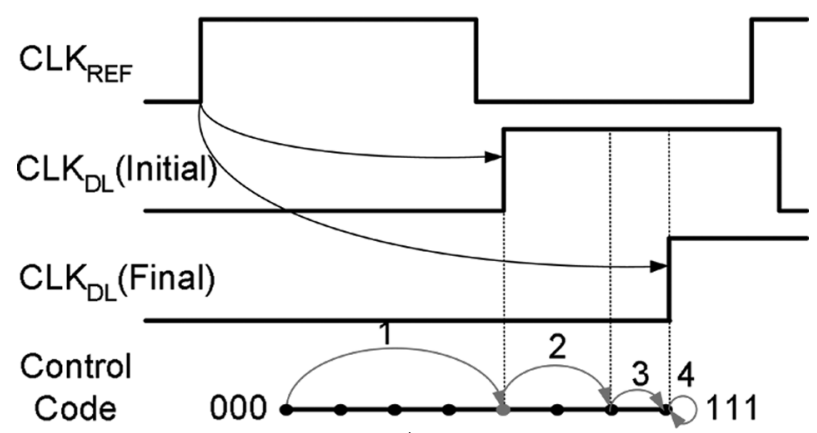

(b)

Fig. 10. Fail-to-lock process for a 3-bit SAR controller when the final delay locks at (a) the minimal delay and (b) the maximal delay.

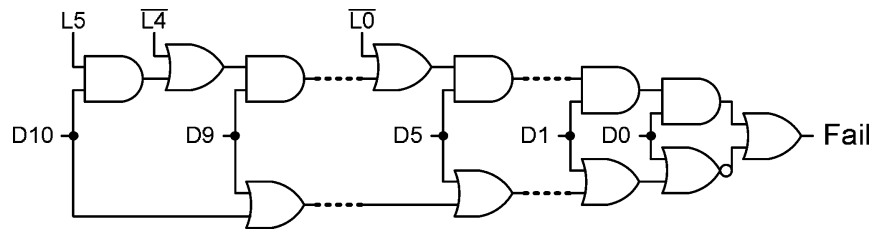

Fig. 11. Fail-to-lock judgment circuit.

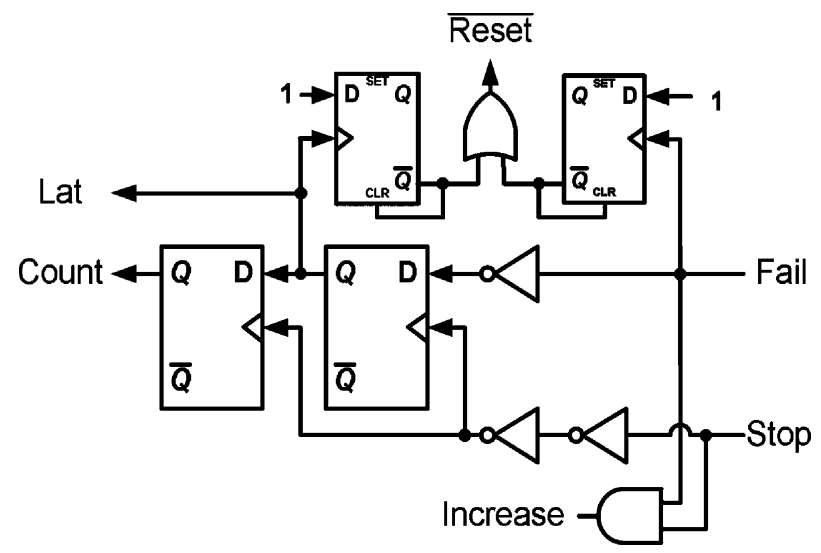

Fig. 12. Timing controller.

Furthermore, since the LDL is controlled by the thermometer code, several binary-to-thermometer decoders are needed and may occupy a large chip area when the number of bits is large. Also, the large number of cascaded LDUs may raise the difficulty of the layout. To enlarge the tunable range without using a large decoder, a nested lattice delay line (NLDL) is adopted. By inserting an LDL between two LDUs, the delay range and 

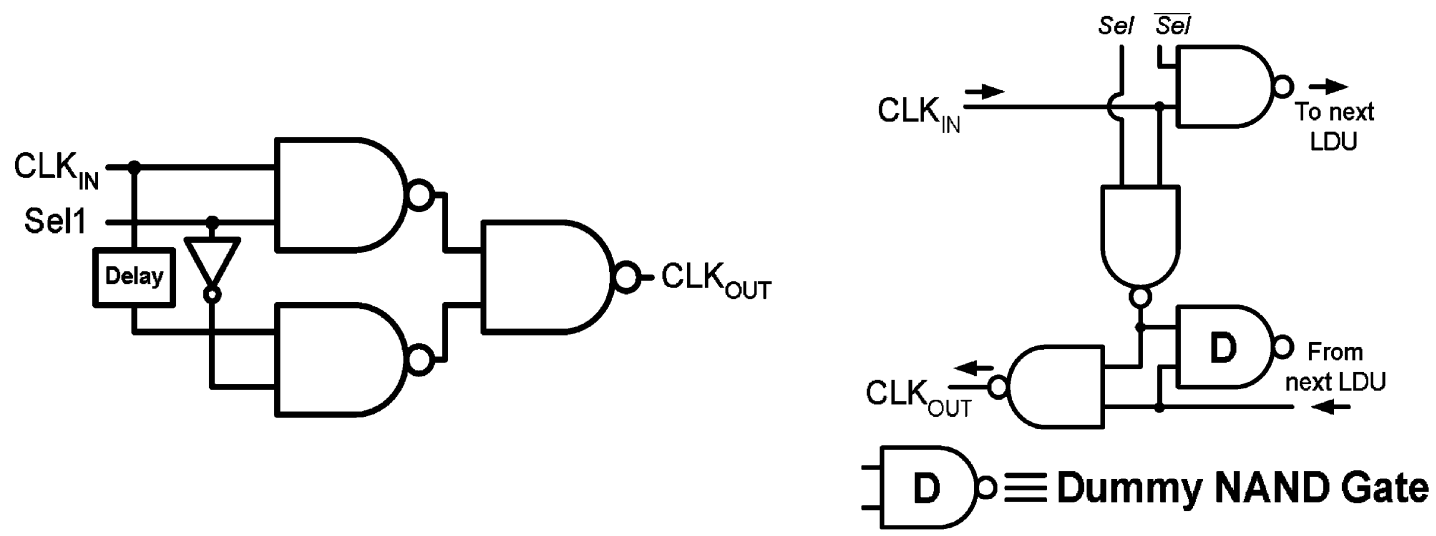

(a)

(b)

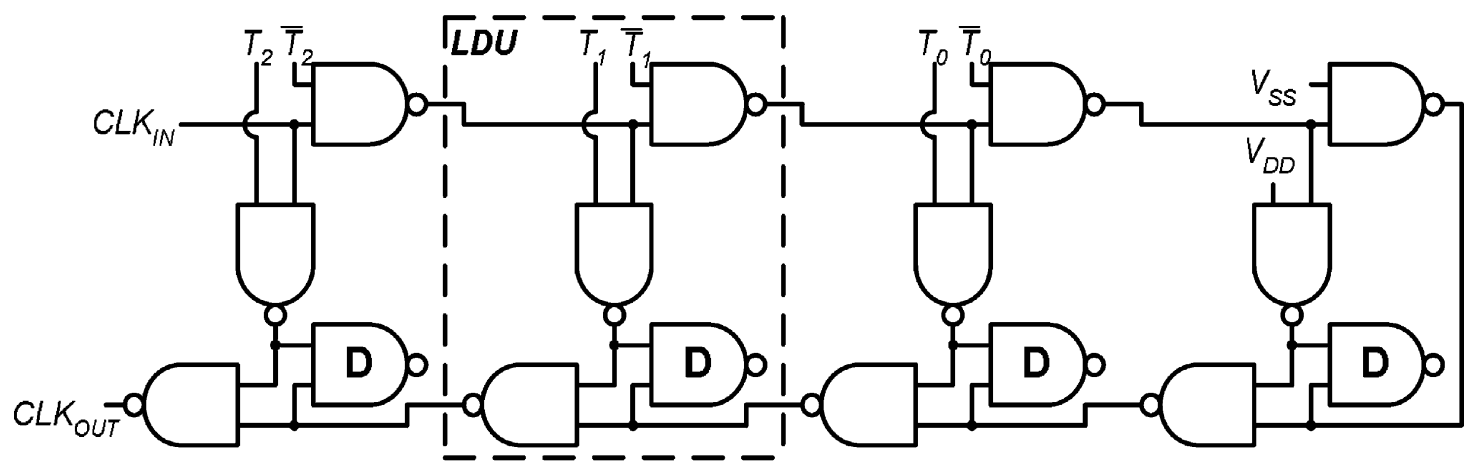

(c)

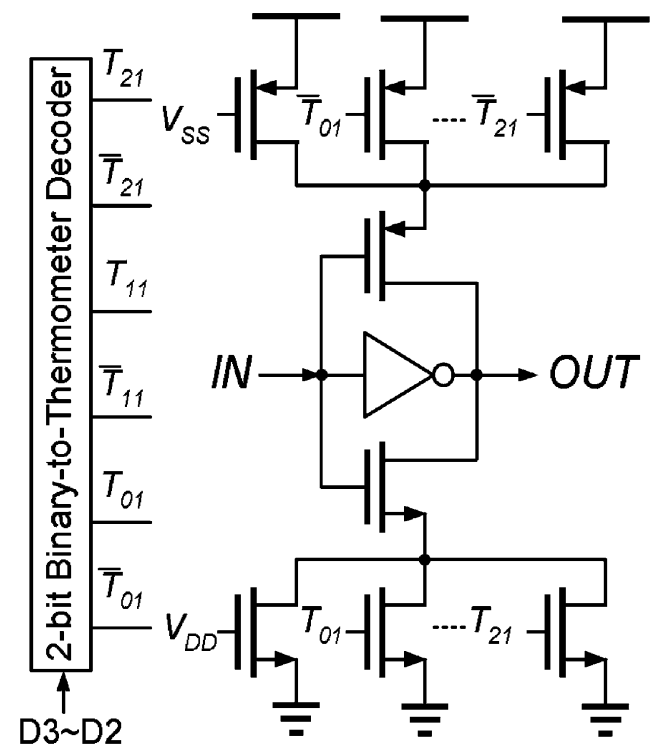

(d)

Fig. 13. (a) Conventional digital delay unit. (b) LDU. (c) LDL. (d) Fine-tuning delay unit.

the delay step are magnified. A current-starving inverter and a fixed inverter in parallel compose the fine delay unit (FDU) to increase the delay resolution as illustrated in Fig. 13(d). The complete DCDL is shown in Fig. 14.

The rising and falling times of the LDU are not equal due to the different driving capabilities of transistors. Hence, the delays through even-numbered and odd-numbered LDUs are not equal.
The conventional solution [6] adds the compensation inverters in the delay units. However the delay resolution is lowered. And the delay of the same LDU varies when the clock goes through different input node of a NAND gate. To alleviate the delay mismatches between different codes, the configuration of the LDL is specially arranged as shown in Fig. 15(a). Note that the label 1 on a NAND gate indicates the input terminal that is more closed 


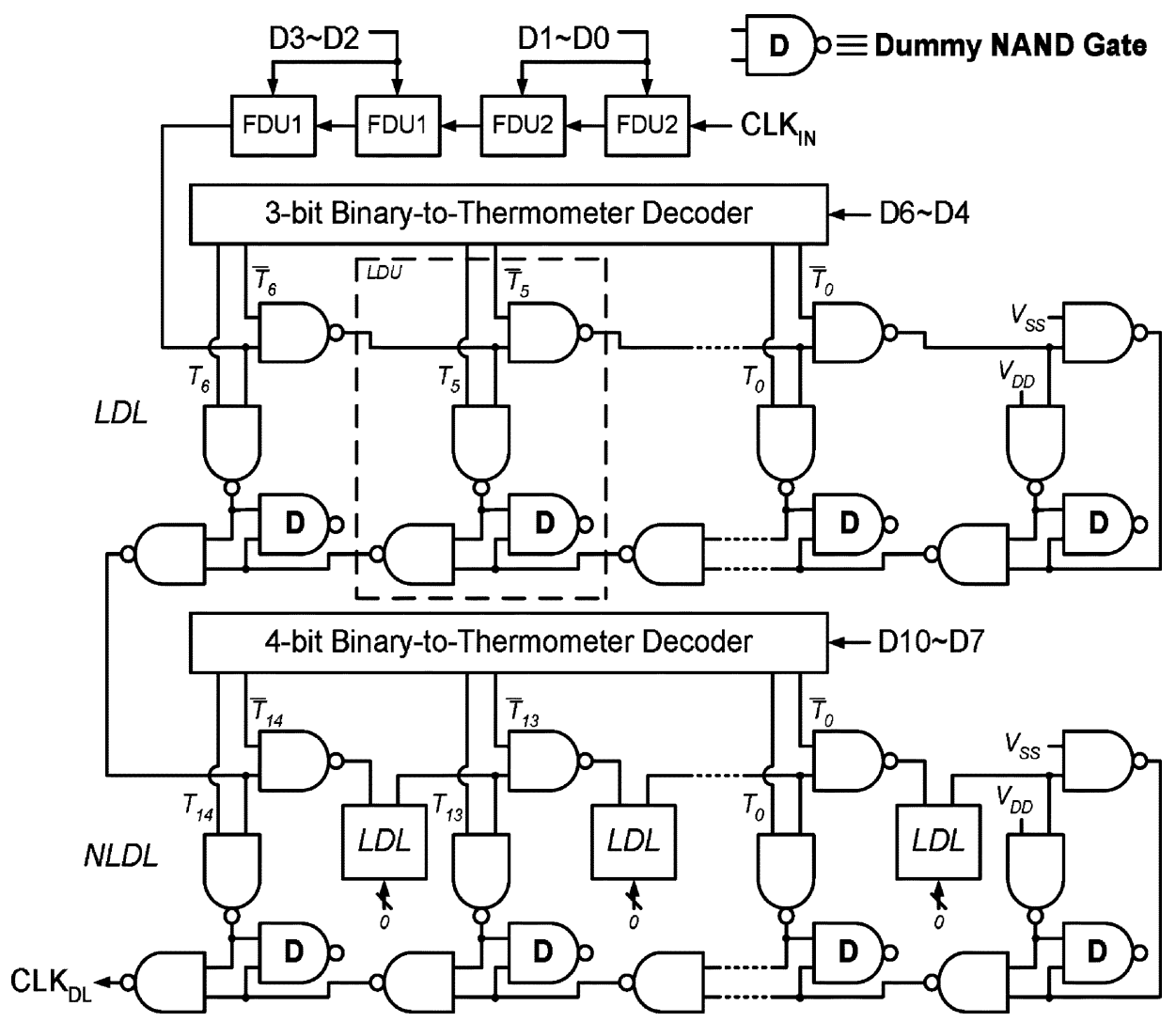

Fig. 14. Digital-controlled delay line.

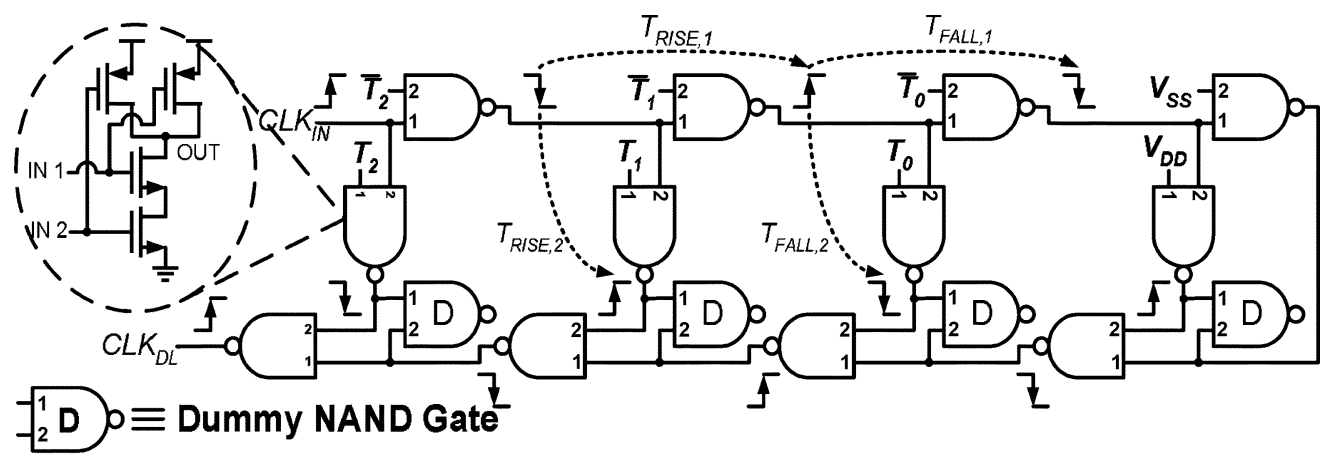

(a)

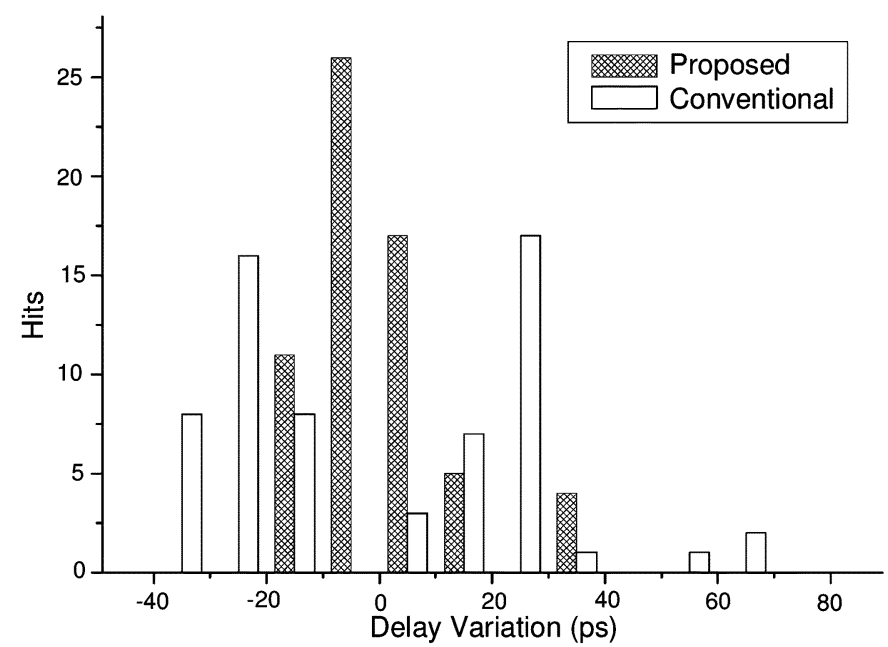

(b)

Fig. 15. (a) Topology of the delay unit for the linearization of delay profile. (b) Simulation result of delay variation. 

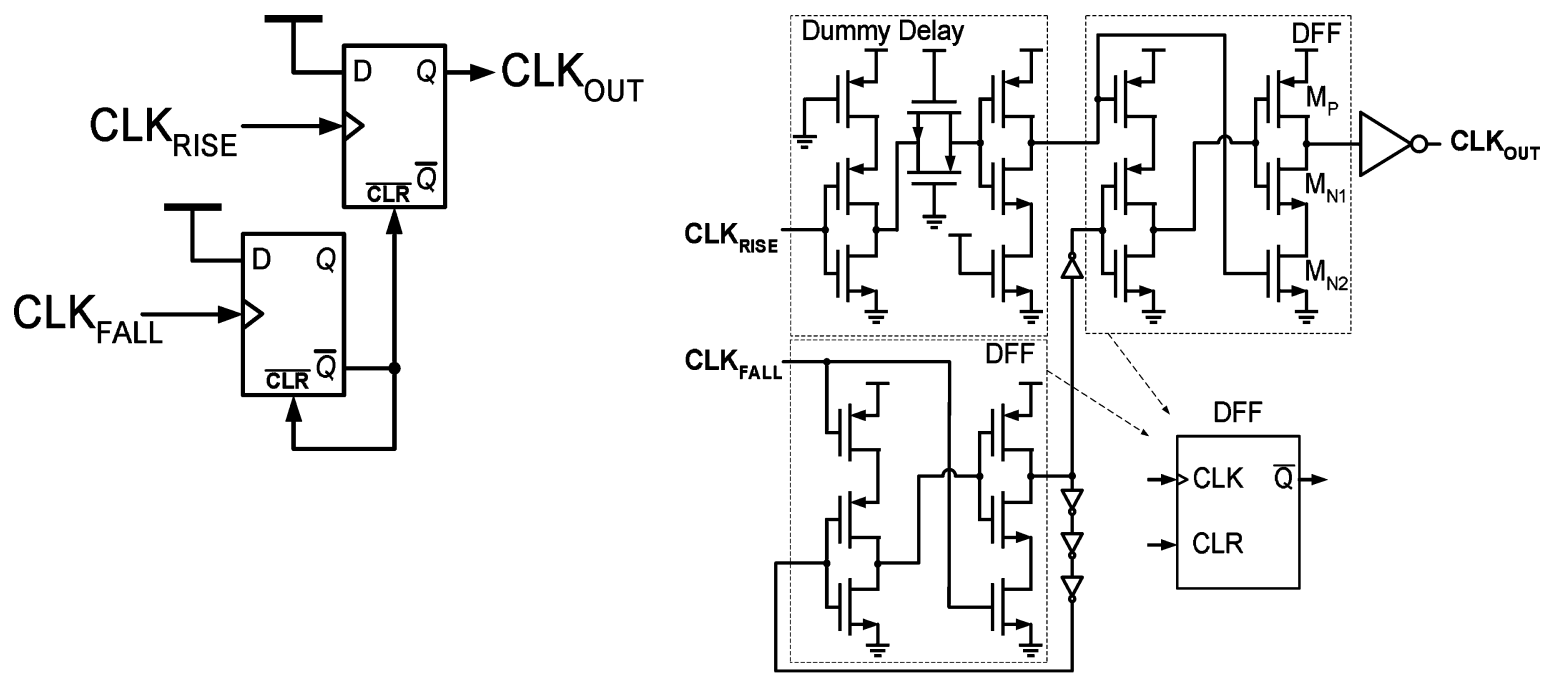

(a)

(b)

Fig. 16. (a) Conventional edge combiner. (b) Proposed edge combiner with balanced path delay.

to the output terminal. In the configuration, the delays for different codes are expressed as

$$
\begin{aligned}
T_{111}= & T_{\mathrm{FALL}, 2}+T_{\mathrm{RISE}, 2} \\
T_{011}= & T_{\mathrm{FALL}, 1}+T_{\mathrm{RISE}, 2}+T_{\mathrm{FALL}, 2}+T_{\mathrm{RISE}, 1} \\
T_{001}= & T_{\mathrm{FALL}, 1}+T_{\mathrm{RISE}, 1}+T_{\mathrm{FAL}, 2}+T_{\mathrm{RISE}, 2} \\
& +T_{\mathrm{FALL}, 1}+T_{\mathrm{RISE}, 1} \\
T_{000}= & T_{\mathrm{FALL}, 1}+T_{\mathrm{RISE}, 1}+T_{\mathrm{FALL}, 1}+T_{\mathrm{RISE}, 2} \\
& +T_{\mathrm{FALL}, 2}+T_{\mathrm{RISE}, 1}+T_{\mathrm{FALL}, 1}+T_{\mathrm{RISE}, 1}
\end{aligned}
$$

where the subscripts 000, 001, 011 and 111 indicate a 3-digit thermometer code and $T_{000}$ and $T_{111}$ depict the maximum and minimum delays of the 4-stage LDL. The subscripts RISE and FALL represent the delays for the rising and the falling clocks and the subscripts 1 and 2 indicate the terminals IN1 and IN2 of a NAND gate, respectively. The delay step of $T_{\mathrm{RISE}, 1}+T_{\mathrm{FALL}, 1}$ is fixed. Several dummy NAND gates in Fig. 15(a) are used to match the loading effect and the numbers of fan-in and fan-out. Fig. 15(b) gives the simulated result for the delay variation. The variation of the proposed configuration is only half in comparison with that of the conventional one.

\section{Edge Combiner}

The conventional edge combiner for the set/reset architecture is implemented by two DFFs as exhibited in Fig. 16(a). The propagation delay from CLK FALL to CLK that from CLK $\mathrm{RISE}_{\text {to }}$ CLK $\mathrm{OUT}$ due to the non-zero reset time of a DFF. The unequal propagation delays distort the accuracy of the duty cycle especially at high frequency. A balanced edge combiner is proposed in Fig. 16(b) to diminish the distortion. It consists of two DFFs and a dummy delay. The propagation delay from $\mathrm{CLK}_{\mathrm{FALL}}$ to CLK $\mathrm{OUT}$ is

$$
T_{D, C-Q}+T_{D, i n v}+T_{D, \text { reset-Q }}+T_{D, i n v}
$$

where $T_{D, C-Q}$ and $T_{D, \text { reset- } Q}$ are the clock-to- $Q$ delay and the reset-to- $Q$ delay, respectively, of the DFF, and $T_{D, i n v}$ is the delay of an inverter. The dummy delay is realized by the same

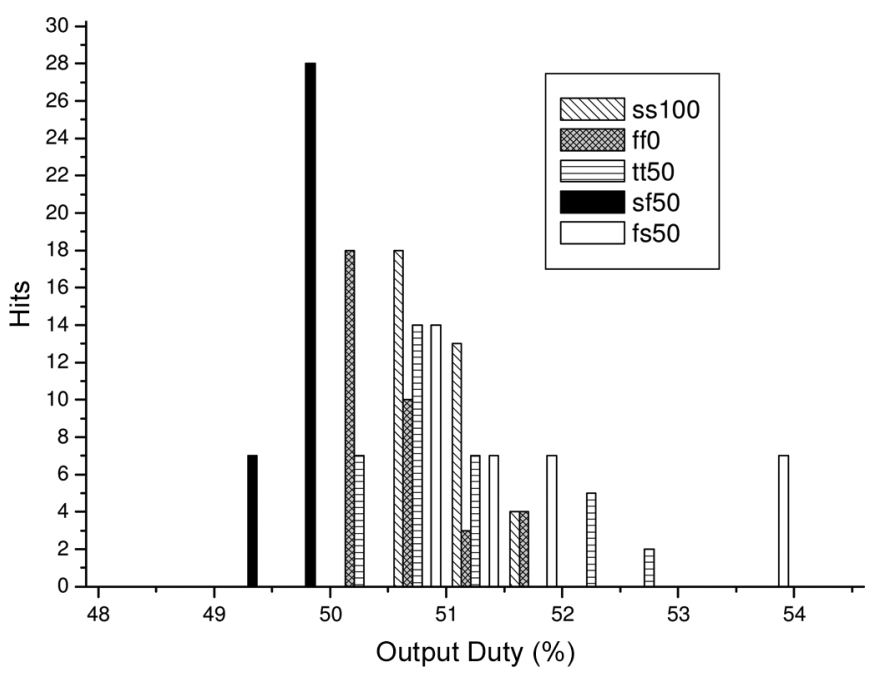

Fig. 17. Simulated result of the proposed edge combiner for different process and temperature variations and input clock duty cycle is from $20 \%$ to $80 \%$ at $500 \mathrm{MHz}$.

DFF and a transmission gate with the same size as the inverter. If the delays of the transmission gate and the inverter are matched, the total propagation delay from CLK $\mathrm{RISE}_{\text {to }} \mathrm{CLK}_{\mathrm{OUT}}$ is

$$
T_{D, \text { reset-Q }}+T_{D, i n v}+T_{D, C-Q}+T_{D, i n v} .
$$

It is the same as that from CLK $\mathrm{FALL}_{\text {to }}$ CLK $\mathrm{OUU}_{\mathrm{OUT}}$. Although the path delays are matched, the output duty cycle is still affected by the discharging capability of $M_{N 1}$ in series with $M_{N 2}$ and the charging capability of $M_{P}$. Fig. 17 manifests the simulation result for different process and temperature variations while the input clock duty cycle is from $20 \%$ to $80 \%$ at $500 \mathrm{MHz}$. The variation of the duty cycle is from $-1 \%$ to $4 \%$.

\section{E. Phase Detector}

A conventional phase detector can be a simple DFF with a binary output. The control code changes back and forth between two or three different values even if the DLL is locked. To avoid this phenomenon, the phase detector in this work is realized by 


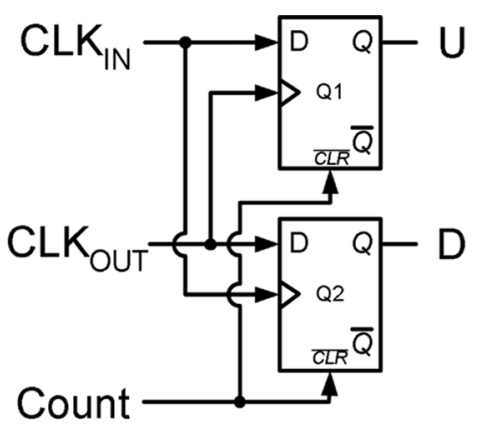

(a)
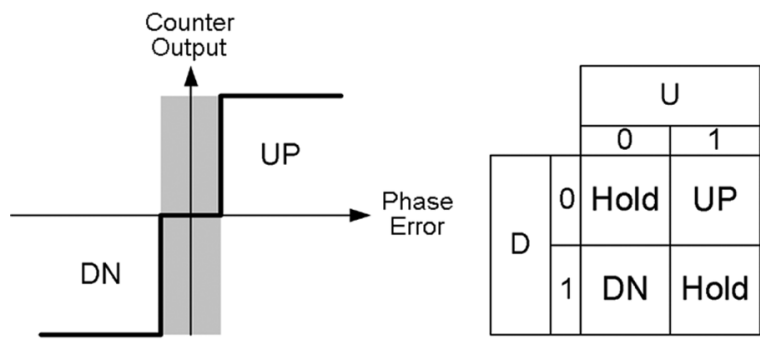

Hold

(b)

Fig. 18. (a) Phase detector. (b) Corresponding transfer characteristic.

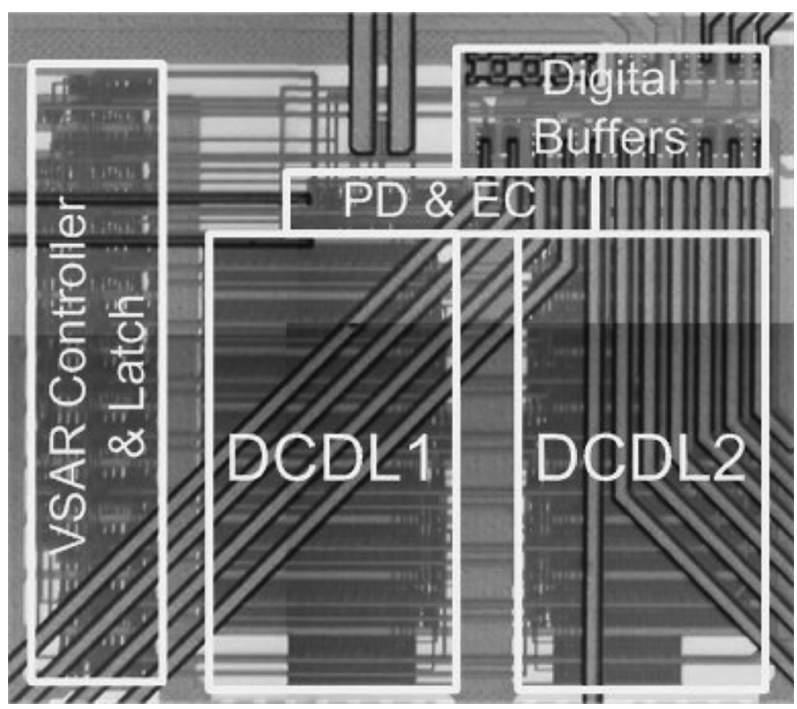

Fig. 19. Die photograph.

two DFFs as shown in Fig. 18(a). The $\mathrm{CLK}_{\mathrm{IN}}$ and CLK $\mathrm{OUT}$ are sampled by each other with these two DFFs. After the VSAR controller is transformed into a counter, the control code changes when only one of the signals $U$ and $D$ is logic one. The counter stops if the signals $U$ and $D$ are the same. The corresponding transfer characteristic of the PD is illustrated in Fig. 18(b). The width of the hold resign is determined by the summation of the offsets for both DFFs and is around 20 ps by simulation.

\section{EXPERIMENTAL RESULTS}

Fig. 19 shows the microphotograph of the proposed ADDLL circuit in $0.18 \mu \mathrm{m}$ CMOS technology. It occupies an active area of $0.5 \mathrm{~mm}$ by $0.4 \mathrm{~mm}$ and consumes $5.4 \mathrm{~mW}$ and $12.6 \mathrm{~mW}$ at $40 \mathrm{MHz}$ and $550 \mathrm{MHz}$, respectively. Fig. 20(a) and (b) illustrates the measured waveforms at $40 \mathrm{MHz}$ and $550 \mathrm{MHz}$ and the output duty cycles are close to $50 \%$. The root-mean-square

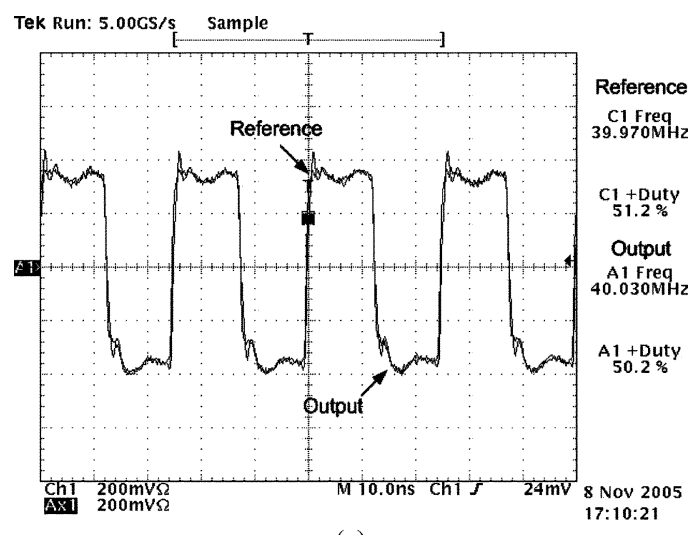

(a)

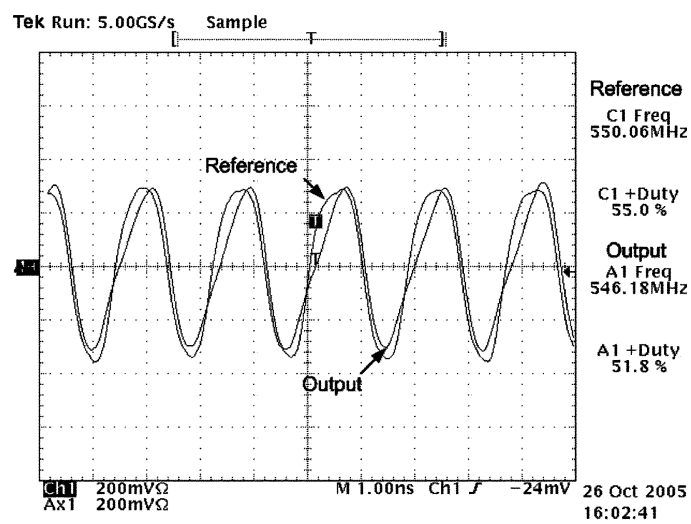

(b)

Fig. 20. Measured waveforms at (a) $40 \mathrm{MHz}$ and (b) $550 \mathrm{MHz}$.

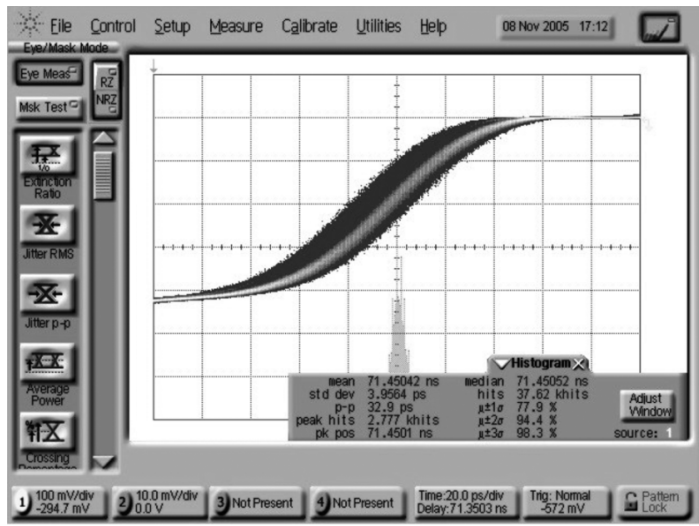

(a)

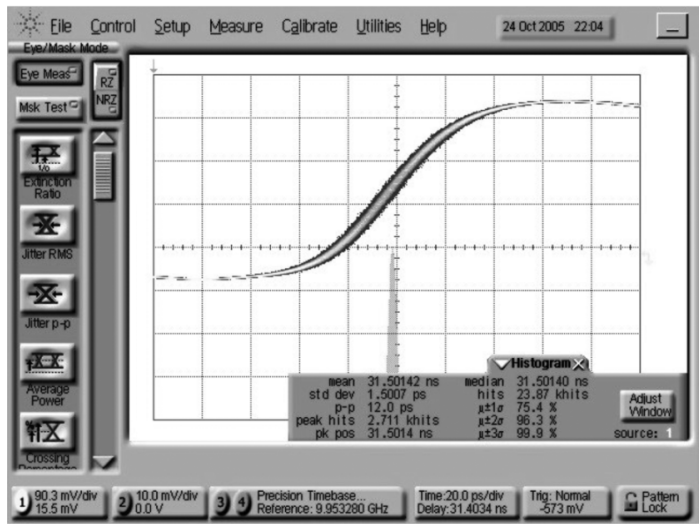

(b)

Fig. 21. Measured jitter histograms at (a) $40 \mathrm{MHz}$ and (b) $550 \mathrm{MHz}$. 


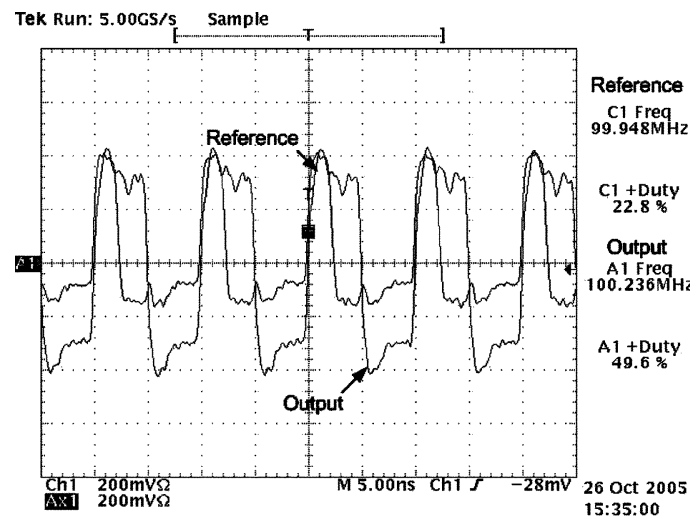

(a)

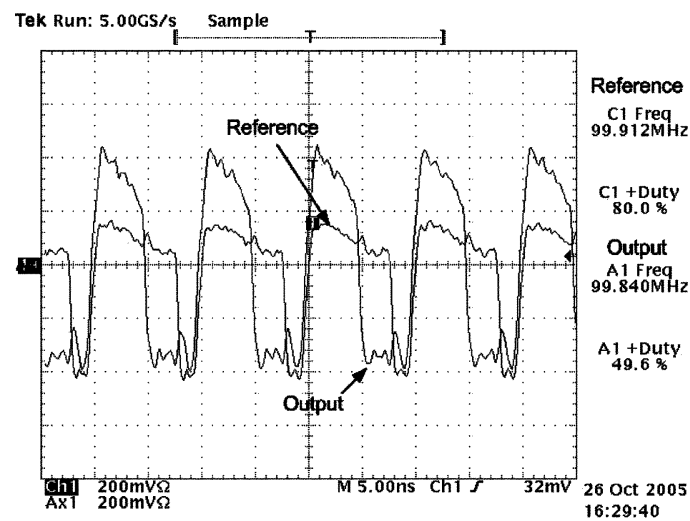

(b)

Fig. 22. Measured result at $100 \mathrm{MHz}$ when the input clock duty cycle is (a) $20 \%$ and (b) $80 \%$.

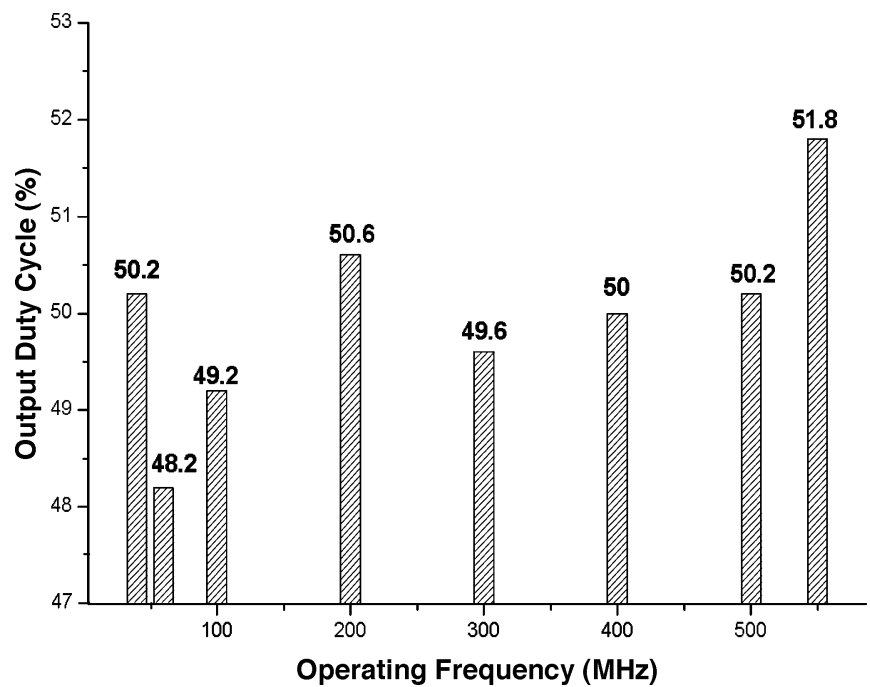

Fig. 23. Measured duty cycles of the output clock in function of operating frequency.

and peak-to-peak jitters at $40 \mathrm{MHz}$ are $3.96 \mathrm{ps}$ and $32.9 \mathrm{ps}$, respectively, while those jitters at $550 \mathrm{MHz}$ are $1.5 \mathrm{ps}$ and $12 \mathrm{ps,}$ respectively, as shown in Fig. 21(a) and (b). Fig. 22(a) and (b) demonstrate the output clock at $100 \mathrm{MHz}$ when the input duty cycles are $20 \%$ and $80 \%$, respectively. The proposed ADDLL still locks correctly and outputs a synchronous clock with $50 \%$ duty cycle. The inverter type output buffer drives the $50 \Omega$ transmission line via an AC-coupled capacitor. The effective bandwidth of the input clock with the duty cycles of $20 \%$ and $80 \%$ is higher

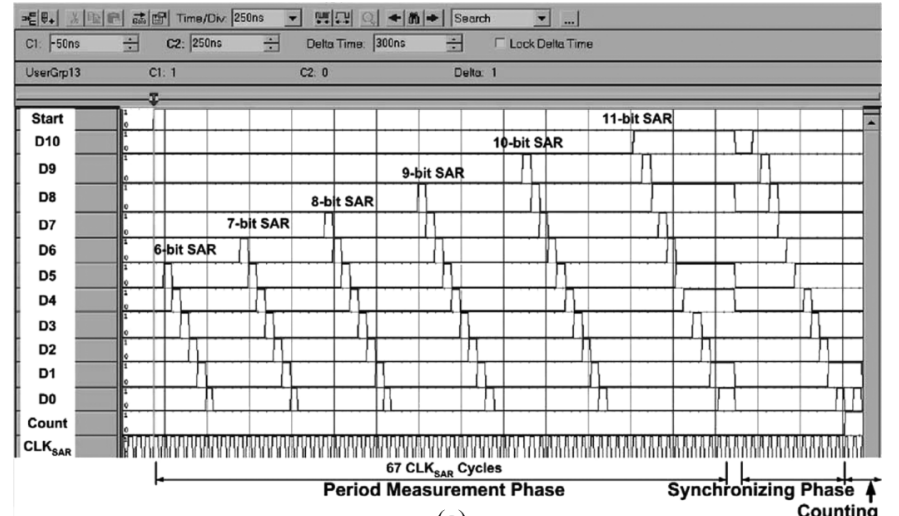

(a)

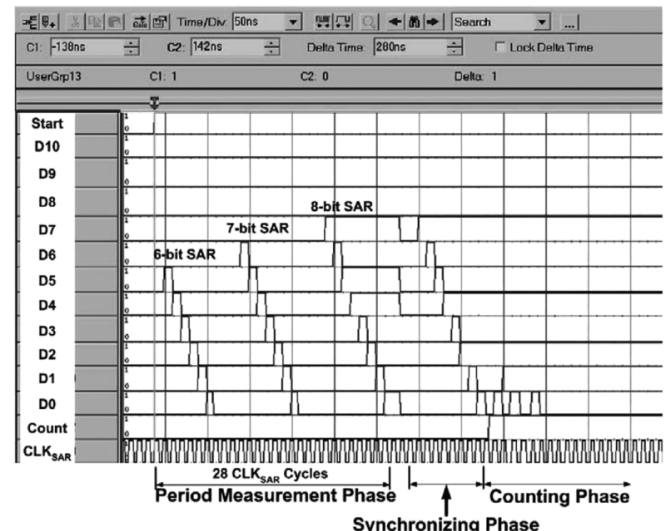

(b)

Fig. 24. Measured digital transient response for the VSAR algorithm at (a) $40 \mathrm{MHz}$ and (b) $200 \mathrm{MHz}$.

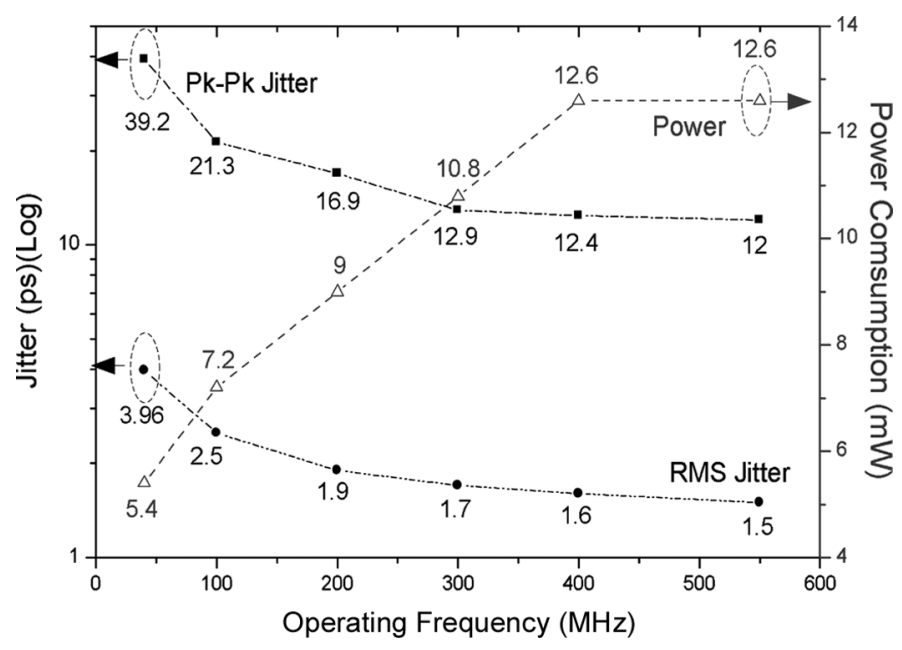

Fig. 25. Measured jitters and power in function of operating frequency.

than the output clock. Hence, the input clock suffers more attenuation than the output clock because of the insufficient bandwidth of the inverter buffers. The measured output duty cycles in a function of the operating frequencies from $40 \mathrm{MHz}$ to $550 \mathrm{MHz}$ is illustrated in Fig. 23. The variation of the duty cycle is within $\pm 2 \%$. The digital transient responses at $40 \mathrm{MHz}$ and $200 \mathrm{MHz}$ are shown in Fig. 24 to manifest the operation of the VSAR algorithm. In the period measurement phase, the number of used bits in binary search increases four times until the delay is long enough for current clock period. In the synchronizing phase, the 
TABLE I

PERFORMANCE SUMMARY AND COMPARISONS

\begin{tabular}{|c|c|c|c|c|c|}
\hline & [7] & [11] & {$[16]$} & [17] & This Work \\
\hline Technology & $\begin{array}{c}0.13 \mu \mathrm{m} 3 \mathrm{M} \\
\text { CMOS }\end{array}$ & $\begin{array}{c}0.18 \mu \mathrm{m} 1 \mathrm{P} 6 \mathrm{M} \\
\mathrm{CMOS}\end{array}$ & $\begin{array}{c}0.18 \mu \mathrm{m} 1 \mathrm{P} 5 \mathrm{M} \\
\mathrm{CMOS}\end{array}$ & $\begin{array}{l}0.18 \mu \mathrm{m} \\
\text { CMOS }\end{array}$ & $\begin{array}{c}0.18 \mu \mathrm{m} 1 \mathrm{P} 6 \mathrm{M} \\
\mathrm{CMOS}\end{array}$ \\
\hline Category & Digital & Digital & $\begin{array}{c}\text { Analog } \\
\text { Dual-loop }\end{array}$ & $\begin{array}{c}\text { Analog } \\
\text { Dual-loop }\end{array}$ & Digital \\
\hline Supply & $\begin{array}{c}1.6 \mathrm{~V} \\
\text { (regulated) }\end{array}$ & $1.8 \mathrm{~V}$ & $1.8 \mathrm{~V}$ & $1.8 \mathrm{~V}$ & $1.8 \mathrm{~V}$ \\
\hline Active Area & $\mathrm{X}$ & $\sim 0.75 \mathrm{~mm}^{2}$ & $0.189 \mathrm{~mm}^{2}$ & $0.046 \mathrm{~mm}^{2}$ & $0.2 \mathrm{~mm}^{2}$ \\
\hline Power & $\begin{array}{c}29 \mathrm{~mW} \\
@ 266 \mathrm{MHz}\end{array}$ & $23 \mathrm{~mW}$ & 63mW@700MHz & $\begin{array}{l}1.2 \mathrm{~mW} @ 250 \mathrm{MHz} \\
6.4 \mathrm{~mW} @ 2 \mathrm{GHz}\end{array}$ & $\begin{array}{c}5.4 \mathrm{~mW} @ 40 \mathrm{MHz} \\
9 \mathrm{~mW} @ 200 \mathrm{MHz} \\
12.6 \mathrm{~mW} @ 550 \mathrm{MHz}\end{array}$ \\
\hline Frequency & $66 \sim 500 \mathrm{MHz}$ & $2-700 \mathrm{MHz}$ & $60 \sim 760 \mathrm{MHz}$ & $250 \mathrm{MHz} \sim 2 \mathrm{GHz}$ & $40 \sim 550 \mathrm{MHz}$ \\
\hline $\begin{array}{l}\text { Wide } \\
\text { Range } \\
\text { Strategy }\end{array}$ & Coarse-Fine & Cyclic-Control & $\begin{array}{l}\text { Multi-phase } \\
\text { Selection }\end{array}$ & $\begin{array}{l}\text { Multi-phase } \\
\text { Selection }\end{array}$ & Coarse-Fine \\
\hline $50 \%$ Duty & Yes & Yes & Yes & $x$ & Yes \\
\hline $\begin{array}{c}\text { DCC } \\
\text { Strategy }\end{array}$ & $\begin{array}{l}\text { Dual-loop } \\
\text { Set/Reset }\end{array}$ & Dual-Structure & $\mathrm{x}$ & $N / A$ & $\begin{array}{l}\text { Single-loop } \\
\text { Set/Reset }\end{array}$ \\
\hline $\begin{array}{c}\text { Delay } \\
\text { Resolution }\end{array}$ & $14 p s$ & $<28 \mathrm{ps}$ & $N / A$ & N/A & $10 \mathrm{ps}$ \\
\hline Lock Time & $\mathrm{X}$ & 32 cycles & $\mathrm{N} / \mathrm{A}$ & $\mathrm{N} / \mathrm{A}$ & 134 14cycles \\
\hline $\begin{array}{l}\text { R.M.S. } \\
\text { Jitter }\end{array}$ & $\mathrm{x}$ & $\begin{array}{l}\text { 42.3ps@2MHz } \\
2 \mathrm{ps@700MHz}\end{array}$ & 5ps@700MHz & $\begin{array}{l}5.25 \mathrm{ps} @ 250 \mathrm{MHz} \\
1.57 \mathrm{ps} @ 1 \mathrm{GHz} \\
2.81 \mathrm{ps} @ 2 \mathrm{GHz} \\
\end{array}$ & $\begin{array}{l}\text { 3.96ps@40MHz } \\
1.9 \mathrm{ps@200MHz} \\
1.5 \mathrm{ps@550MHz}\end{array}$ \\
\hline Pk-Pk Jitter & $x$ & $\begin{array}{c}\text { 310ps@2MHz } \\
\text { 17.6ps@700MHz }\end{array}$ & 28ps@700MHz & $\begin{array}{l}31.6 \mathrm{ps} @ 250 \mathrm{MHz} \\
\text { 10.7ps@1GHz } \\
20.4 \mathrm{ps} @ 2 \mathrm{GHz}\end{array}$ & $\begin{array}{c}32.9 \mathrm{ps} @ 40 \mathrm{MHz} \\
16.9 \mathrm{ps} @ 200 \mathrm{MHz} \\
12 \mathrm{ps} @ 550 \mathrm{MHz}\end{array}$ \\
\hline
\end{tabular}

$\mathrm{X}$ : Not mentioned. N/A: Not available.

clock skew contributed by the edge combiner is compensated. The closed-loop characteristic is also guaranteed after the VSAR algorithm completes. Fig. 25 shows the measured jitter and power consumption for different operating frequencies. Table I gives the performance summary and comparisons with previous works.

\section{CONCLUSION}

A $40-550 \mathrm{MHz}$, all-digital delay-locked loop has been fabricated in $0.18 \mu \mathrm{m}$ CMOS technology. The proposed VSAR algorithm avoids the harmonic-locking issue and reduces the lock time in a wide-range operation. With the aid of the proposed set/reset control scheme, the nested lattice delay line and the balanced edge combiner, the ADDLL can achieve low power, small area and fast lock with output duty cycle close to $50 \%$ while the input duty cycle varies.

\section{ACKNOWLEDGMENT}

The authors would like to thank the Chip Implementation Center (CIC) of the National Science Council, Taiwan, for fabricating the chips.

\section{REFERENCES}

[1] A. Alvandpour, R. K. Krishnamurthy, D. Eckerbert, S. Apperson, B. Bloechel, and S. Borkar, "A $3.5 \mathrm{GHz} 32 \mathrm{~mW} 150 \mathrm{~nm}$ multiphase clock generator for high-performance microprocessors," in IEEE ISSCC Dig. Tech. Papers, 2003, pp. 112-113, 489.

[2] K. Nose, A. Shibayama, H. Kodama, M. Mizuno, M. Edahiro, and N. Nishi, "Deterministic inter-core synchronization with periodically all-in-phase clocking for low-power multi-core SoCs," in IEEE ISSCC Dig. Tech. Papers, 2005, pp. 296-297, 599.
[3] J. R. Burnham, G. K. Yeh, E. Sun, and C.-K. Yang, "Design and analysis of a jitter-tolerant digital delay-locked-loop based fraction-ofclock delay line," in IEEE ISSCC Dig. Tech. Papers, 2004, pp. 352-353, 532.

[4] J.-T. Kwak, C.-K. Kwon, K.-W. Kim, S.-H. Lee, and J.-S. Kih, "Low cost high performance register-controlled digital DLL for $1 \mathrm{Gbps}$ X32 DDR SDRAM," in Symp. VLSI Circuits Dig. Tech. Papers, 2003, pp. 283-284.

[5] T. Hamamoto, K. Furutani, T. Kubo, S. Kawasaki, H. Iga, T. Kono, Y. Konishi, and T. Yoshihara, "A 667-Mb/s operating digital DLL architecture for 512-Mb DDR SDRAM," IEEE J. Solid-State Circuits, vol. 39, no. 1, pp. 194-206, Jan. 2004.

[6] Y.-J. Jeon, J.-H. Lee, H.-C. Lee, K.-W. Jin, K.-S. Min, J.-Y. Chung, and H.-J. Park, "A 66-333-MHz 12-mW register-controlled DLL with a single delay line and adaptive-duty-cycle clock dividers for production DDR SDRAMs," IEEE J. Solid-State Circuits, vol. 39, no. 11, pp. 2087-2092, Nov. 2004.

[7] T. Matano, Y. Takai, T. Takahashi, Y. Sakito, I. Fujii, Y. Takaishi, H. Fujisawa, S. Kubouchi, S. Narui, K. Arai, M. Morino, M. Nakamura, S. Miyatake, T. Sekiguchi, and K. Koyama, "A 1-Gb/s/pin 512-Mb DDRII SDRAM using a digital DLL and a slew-rate-controlled output buffer," IEEE J. Solid-State Circuits, vol. 38, no. 5, pp. 762-768, May 2003.

[8] K. Minami, M. Miruno, H. Yamaguchi, T. Nakano, Y. Matsushima, Y. Sumi, T. Sato, H. Yamashida, and M. Yamashina, "A $1 \mathrm{GHz}$ portable digital delay-locked loop with infinite phase capture ranges," in IEEE ISSCC Dig. Tech. Papers, 2000, pp. 350-351, 469.

[9] K.-H. Cheng, Y.-L. Lo, and W.-F. Yu, "A mixed-mode delay-locked loop for wide-range operation and multiphase outputs," in Proc. IEEE Int. Symp. Circuits and Systems (ISCAS), 2003, vol. II, pp. 25-28.

[10] A. Rossi and G. Fucilli, "Nonredundant successive approximation register for A/D converters," Electron. Lett., vol. 32, no. 12, pp. 1055-1057, Jun. 1996.

[11] H. H. Chang and S. I. Liu, "A wide-range and fast-locking all-digital cycle-controlled delay-locked loop," IEEE J. Solid-State Circuits, vol. 40, no. 3, pp. 661-670, Mar. 2005. 
[12] R.-J. Yang and S.-I. Liu, "A wide-range multiphase delay-locked loop using mixed-mode VCDLs," IEICE Trans. Electron., vol. E88-C, pp. 1248-1252, Jun. 2005.

[13] K. Nakamura, M. Fukaishi, Y. Hirota, Y. Nakazawa, and M. Yotsuyanagi, "A CMOS 50\% duty cycle repeater using complementary phase blending," in Symp. VLSI Circuits Dig. Tech. Papers, 2000, pp. $48-49$.

[14] G.-K. Dehng, J.-W. Lin, and S.-I. Liu, "A fast-lock mixed-mode DLL using a 2-b SAR algorithm," IEEE J. Solid-State Circuits, vol. 36, no. 10, pp. 1464-1471, Oct. 2001.

[15] C.-K. Liang, R.-J. Yang, and S.-I. Liu, "An all-digital fast-locking programmable DLL-based clock generator," IEEE J. Solid-State Circuits, submitted for publication.

[16] S.-J. Bae, H.-J. Chi, Y.-S. Sohn, and H.-J. Park, "A VCDL-based 60-760-MHz dual-loop DLL with infinite phase-shift capability and adaptive-bandwidth scheme," IEEE J. Solid-State Circuits, vol. 40, no. 5, pp. 1119-1129, May 2005.

[17] B.-G. Kim and L.-S. Kim, "A 250-MHz-2-GHz wide-range delaylocked loop," IEEE J. Solid-State Circuits, vol. 40, no. 6, pp. 1310-1321, Jun. 2005.

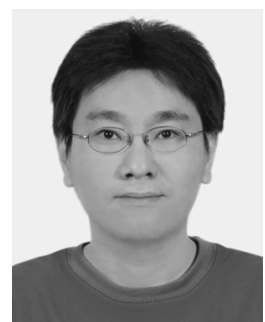

Rong-Jyi Yang (S'03) was born in Taipei, Taiwan, R.O.C., in 1973. He received the B.S. degree in electrical engineering from National Central University, Jhongli, Taiwan, R.O.C., in 1998, and the Ph.D. degree from National Taiwan University, Taipei, in 2006.

Since August 2006, he has been an Assistant Professor in the Department of Electrical Engineering, Chung Gung University, Tao-Yuan, Taiwan. His research interests include both analog and digital approaches of phase-locked loops, delay-locked loops, and high-speed CMOS data-communication circuits for multiple gigabit applications.

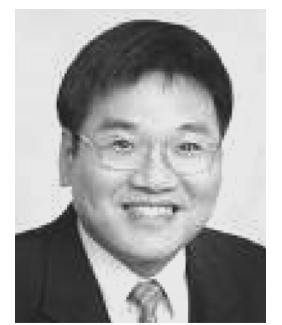

Shen-Iuan Liu (S'88-M'93-SM'03) was born in Keelung, Taiwan, R.O.C., in 1965. He received the B.S. and Ph.D. degrees in electrical engineering from National Taiwan University (NTU), Taipei, Taiwan, in 1987 and 1991, respectively.

During 1991-1993, he served as a Second Lieutenant in the Chinese Air Force. During 1991-1994, he was an Associate Professor in the Department of Electronic Engineering of National Taiwan Institute of Technology. He joined the Department of Electrical Engineering, NTU, in 1994 and he has been a Professor since 1998. His research interests are in analog and digital integrated circuits and systems.

Dr. Liu has served as Chair of the IEEE SSCS Taipei Chapter since 2004. He has served as General Chair of the 15th VLSI Design/CAD Symposium, Taiwan, 2004 and Program Co-chair on the Fourth IEEE Asia-Pacific Conference on Advanced System Integrated Circuits, Japan, 2004. He was the recipient of the Engineering Paper Award from the Chinese Institute of Engineers in 2003, the Young Professor Teaching Award from MXIC Inc., the Research Achievement Award from NTU, and the Outstanding Research Award from National Science Council in 2004. He served as a Technical Program Committee member for A-SSCC and ISSCC in 2005. In 2006, he became an Associate Editor for the IEEE JOURNAL OF SOLID-STATE CIRCUITS and an Associate Editor for the IEEE TRANSACTIONS ON CIRCUITS AND SYSTEMS-II: EXPRESS BRIEFS. $\mathrm{He}$ is a senior member of IEEE and a member of IEICE. 